\title{
Caenorhabditis Elegans NHR-14/HNF4a Cooperates with cep-1/ p53 to Regulate DNA Damage-induced Apoptosis
}

Jianwei Sun ( $\sim$ jianwei5639@163.com )

Yunnan University https://orcid.org/0000-0002-3045-2724

Lei Sang

Yunnan University

Rui Dong

Yunnan University

Rui Liu

Kunming Medical University

Qinggang Hao

Yunnan University

Xingshun Wang

Yunnan University

\section{Yong Zeng}

Kunming Medical University

Mei Yang

Yunnan University

\section{Research Article}

Keywords: NHR-14, CEP-1/p53, DNA damage, apoptosis, Caenorhabditis elegans

Posted Date: January 17th, 2022

DOI: https://doi.org/10.21203/rs.3.rs-1231254/v1

License: (c) (1) This work is licensed under a Creative Commons Attribution 4.0 International License.

Read Full License 

6

Caenorhabditis elegans NHR-14/HNF4 $\alpha$ cooperates with cep-1/ p53 to regulate DNA damage-induced apoptosis

Lei Sang ${ }^{1 \#}$, Rui Dong ${ }^{1 \#}$, Rui Liü ${ }^{2 \#}$, Qinggang Hao ${ }^{1}$, Xingshun Wang ${ }^{1,3}$, Yong, Zeng ${ }^{3}$, Mei Yang $^{1^{*}}$, Jianwei Sun ${ }^{1 *}$

1 Center for Life Sciences, School of Life Sciences, State Key Laboratory for Conservation and Utilization of Bio-Resources in Yunnan, Yunnan University, Kunming, China

2 Clinical Laboratory, the second Affiliated Hospital of Kunming Medical University,

Kunming, China

3 The Sixth Affiliated Hospital of Kunming Medical University, Yuxi, China 2

3 \#These authors contribute equally to this work

4 *Correspondence to Jianwei Sun: jwsun@ynu.edu.cn or Mei Yang: meiyang@ynu.edu.cn 5

6 Lei Sang : $\underline{\text { sangl@mail.ynu.edu.cn }}$

17 Rui Dong: 2451203260@qq.com

8 Rui Liu: $\underline{782738758 @ q q . c o m}$

9 Qinggang Hao: haoqinggang@yeah.net

Xingshun Wang: 11653014@mail.sustech.edu.cn

1 Yong, Zeng: zengyong@ kmmu.edu.cn 

1

\section{Abstract}

Background: Nuclear hormone receptor is involved in transcriptional regulation and many important cellular processes including development and metabolism. However, its role in DNA damage-induced apoptosis remains elusive.

Methods: Synchronized young adult animals were irradiated with different doses of gamma-Ray, and then put back to culture at $20^{\circ} \mathrm{C}$. Germline cell apoptosis was scored at different time point.

Results: Deletion of $n h r-14$ led to decreased DNA damage-induced germline apoptosis, but not the physiological programmed cell death. We also demonstrate that $n h r-14$ is not a checkpoint gene and functions downstream of the checkpoint pathway. Moreover, we show that $n h r-14$ regulates egl-1 and ced-13 transcription upon DNA damage. Mechanistically, NHR-14 and its human homolog hepatocyte nuclear factor 4 transcription factor alpha $(\mathrm{HNF} 4 \alpha)$ form a complex with CEP-1/p53.

Conclusions: Our results indicate that NHR-14/ HNF4 $\alpha$, in addition to its role as a nuclear hormone receptor, also cooperates with CEP-1/p53 to regulate DNA damage-induced apoptosis.

Keywords: NHR-14, CEP-1/p53, DNA damage, apoptosis, Caenorhabditis elegans 


\section{Background}

Nuclear hormone receptors (NHRs) comprise a large family of transcription factors distinguished by a highly conserved DNA binding domain and a structurally conserved ligand-binding domain. There are 284 predicted NHR genes in C.elegans[1]. Nuclear hormone receptors have been shown to regulate important developmental process[2-5]. NHR-14, an estrogenic hormone receptor, has been reported to regulate innate immunity and iron uptake [6]. However, the role of NHR-14 in programmed cell death has not been documented.

Programmed cell death (i.e., apoptosis) is one of the most important processes in the metazoans development. It plays the key roles in animal development and DNA damage repair. DNA damage-induced apoptosis is the cell death happening after severe DNA damage, which is associated with a number of human diseases including cancer. Caenorhabditis elegans has been used extensively to study the programmed cell death induced by DNA damage responses. We previously demonstrated that prmt-5, the C. elegans homolog of mammalian type II protein arginine methyltransferase PRMT5, negatively regulates DNA damage-induced apoptosis[7]. prmt-5(gk357) deletion mutants have increased germline programmed cell death after DNA damage. Furthermore, genetic analyses indicated that prmt-5-mediated apoptosis depends on cep-1/p53 and requires the core cell death pathway. In C. elegans, the p53 homolog CEP-1 acts as a key effector to mediate germ cell apoptosis triggered by ionizing irradiation[8]. Although many factors have been reported to be involved in p53/cep-1 dependent apoptotic pathway, the details of this pathway are not completely understood yet.

In the present study, we show that RNAi knockdown of $n h r-14$ suppresses DNA damage-induced apoptosis in prmt-5(gk357) deletion mutants. Further, we show that $n h r-14$ is a new factor involved in the DNA damage-induced apoptosis and that $n h r-14$ is not a checkpoint gene and functions downstream of the checkpoint genes. Our study confirmed that NHR-14 cooperates with CEP-1/p53 to regulate egl-1 (Bcl-2 homology region 3 domain containing gene) and ced-13 (Bcl-2 homology region 3 domain containing gene) expression and DNA damage-induced apoptosis, which reveals a novel role and mechanism for NHR-14/HNF4 $\alpha$ in apoptosis and hepatocarcinogenesis. Our study might be of potential 
73 therapeutic value.

74

75 


\section{Methods}

\section{C. elegans Strains and Genetics}

The strains of $n h r-14(t m 1473), b r c-1(t m 1145), v p s-18(t m 1125)$ and $x p f-1(t m 2482)$ were provided by Dr. Shohei Mitani. prmt-5(gk357), cep-1(gk138),gld-1(op236), akt-1(ok525), abl-1(ok171), ced-9(n1653), hus-1(op244), clk-2(mn159) and him-6(ok412) strains were provided by C. elegans Genetic Center (CGC). Worms were cultured and maintained using standard procedures. The Bristol N2 strain was used as wildtype. Deletion strains were outcrossed with N2 strain for 6 times. Double mutants were constructed with standard protocol.

\section{Germ Cell Apoptosis Assay}

Synchronized young adult animals were irradiated with gamma-Ray (120Gy), which was located in the Peking University Health Science Center. Irradiated animals were put back to culture at $20^{\circ} \mathrm{C}$ at different time points. Worms with normal germline morphology were scored for germline cell apoptosis with DIC Zeiss microscope.

\section{Radiation Sensitivity Assay}

N2 worms and nhr-14(tm1473) and hus-1(op244), hus-1(op244);nhr-14(tm1473), clk-2(mn159), clk-2(mn159);nhr-14(tm1473) mutant worms were irradiated at the L4 stage as indicated. Eggs laid 8-24hr after irradiation (corresponding to pachytene-stage germ cells at the time of irradiation) were counted. Unhatched eggs surviving animals were counted for days 1 and 2. The result represents the percent of survival of embryos of six different animals per strain.

\section{Mammalian Cell Culture, Transfection and Immunoprecipitation}

Human embryonic kidney (HEK293) cells were grown in Dulbecco's modified Eagle's medium (HyClone) supplemented with 10\% fetal bovine serum (HyClone). The transfection was performed with $2.0 \mathrm{ug}$ of mammalian vectors expressing worm proteins with different tags (i.e., pCMV-myc-cep-1, pCMV-tag2B-nhr-14) using PEI reagent. After 36h of 
transfection, cells were harvested and lysed in a buffer containing 50mM Tris ( $\mathrm{pH} 8.0)$, $150 \mathrm{mM} \mathrm{NaCl}, 0.5 \%$ sodium deoxycholate, $1 \%$ Triton X-100, 1mM phenylmethylsulfonyl fluoride (PMSF). The lysate was incubated with anti-Flag antibody (M2)-conjugated agarose beads (Sigma) for more than $2 \mathrm{~h}$ at $4^{0} \mathrm{C}$. The beads were washed extensively in a buffer containing 50mM Tris (pH 8.0), 150mM NaCl, $1 \mathrm{mM}$ PMSF and 1\% NP-40. Bound proteins were eluted and resolved on sodium dodecyl sulfate polyacrylamide gel electrophoresis (SDS-PAGE) and detected with Western blot assay.

\section{Western blot assay}

Cells were scraped and lysed in lysis buffer on ice for $15 \mathrm{~min}, 15 \mu \mathrm{g}$ total proteins were loaded on SDS-PAGE Gel as co-immunoprecipitation experiment input. The SDS-PAGE gel first run on $60 \mathrm{~V}$ for $30 \mathrm{~min}$ and then $120 \mathrm{~V}$ until the dye run out of the gel, then the protein was transferred to PVDF membrane. The membranes were blocked in 5\% nonfat dry milk in Tris-buffered saline, $0.05 \%$ Tween for 30 minutes at room temperature, and then incubated with primary antibodies for $2 \sim 4$ hours at $4{ }^{0} \mathrm{C}$, followed by incubation with secondary antibody for $60 \mathrm{~min}$ at RT. The results were detected by an ECL-plus Western blotting detection system (Tanon-5200Multi). The primary antibodies used in this study were as follows: anti-Flag (Sigma, Cat\#:F3165) ; anti-Myc (Sigma, Cat\#:HPA055893); GAPDH (Santa CruZ, Cat\#: sc-32233).

\section{GST Pull-down Assay}

For GST pull-down assay, purified GST or GST- CEP-1 fusion proteins were immobilized on glutathione-Sepharose beads and incubated with ${ }^{[35 S]}$ methionine-labeled NHR-14 at $4^{0} \mathrm{C}$ for more than $2 \mathrm{~h}$. The beads were washed extensively and bound proteins were eluted and separated on $12 \%$ SDS-PAGE and exposed to phospho- imager (Amersham) for autoradiography.

\section{RT-qPCR assay}

Total C.elegans RNA was extracted using TRIZOL method and cDNA was synthesized using iScript cDNA Synthesis Kit (Bio-Rad Laboratories). qPCR was performed in an iCycler 
136 thermocycler (Bio-Rad Laboratories) using iQ SYBR Green Supermix (Bio-Rad

137 Laboratories). mRNA levels were quantified using iCycler software (Bio-Rad Laboratories)

138 and were normalized to $t b g-1$. The primers used for RT-qPCR were as follows:

139 egl-1 q-PCR NS: 5'-gattcttctcaatttgccgacg-3'; egl-1 q-PCR CAS: 5'-tcatctgagcatcgaagtcatc-3';

140 ced-13 q-PCR NS: 5'-acggtgtttgagttgcaagc-3';

141 ced-13 q-PCR CAS: 5'-gtcgtacaagcgtgatggat-3; tbg-lq-PCR NS:

142 5'-cgtcatcagctggtagaaca-3'; tbg-1q-PCR CAS: 5'-tgatgactgtccacgttgga-3'.

143 All experiments were analyzed in triplicates.

144

145 Statistical analysis

146 All the experiments were repeated three times and each experiment was performed in 3 147 replicates per sample. Data were analyzed using SPSS 19.0 and GraphPad Prism 6.0. 148 Student's t-test, Spearman correlation, Kaplan-Meier, log-rank test and Cox regression 149 survival and Statistical significance was defined as $* P<0.05, * * P<0.01$ or $* * * P<0.001$. 150 
Inactivation of $n h r-14 / \mathrm{HNF} 4 \alpha$ inhibits DNA damage-induced apoptosis.

To examine whether nuclear hormone receptor is directly involved in the regulation of DNA damage-induced apoptosis, we performed RNAi screen in the background of prmt-5(gk357). We found that knockdown of $n h r-14 / \mathrm{HNF} 4 \alpha$ RNAi reduced the DNA damage-induced programmed cell death in prmt-5(gk357) (Figure 1A) after ionizing irradiation. Further analysis showed that the C. elegans $n h r-14$ gene is defined by the open reading frame T01B10.4 located on the linkage group $\mathrm{X}$, and encodes a protein of 435 amino acids. $n h r-14(\operatorname{tm} 1473)$ deletion mutant contains a deletion of 409bp in the third exon and third intron of $n h r-14$, and this deletion will result in an early stop of NHR-14 translation[9].

In order to test the function of $n h r-14 / \mathrm{HNF} 4 \alpha$ in DNA damage-induced apoptosis, we used $n h r-14(t m 1473)$ deletion mutants to analyze the germ cell apoptosis after ionizing irradiation, we found that $n h r-14(t m 1473)$ inhibited DNA damage-induced apoptosis in prmt-5(gk357) at different gamma-irradiation doses (Figure 1B) and different times (Figure 1C). In order to rule out that whether the decreased of apoptosis caused by DNA damage in $n h r-14(t m 1473)$ is due to a defect in cell corpse clearance, we analyzed the germ cell apoptosis in ced-1(e1375); prmt-5(gk357) and ced-1(e1375); prmt-5(gk357); nhr-14(tm1473), the results showed that $n h r-14(t m 1473)$ still significantly inhibited DNA damage-induced apoptosis in prmt-5(gk357) in the background of ced-1(e1735) (Figure 1D). Our results suggested that $n h r-14$ functions downstream of prmt-5 and regulates DNA damage-induced programmed cell death.

To further determine whether $n h r-14 / \mathrm{HNF} 4 \alpha$ is a new factor involved in the DNA damage-induced cell apoptosis, we performed epistasis analysis using several well-defined cell survival molecules including AKT-1/AKT, ABL-1/ABL and CED-9/BCL2. Previous studies have demonstrated that loss-of-function mutation of C. elegans akt-1(ok525) exhibits dramatically increased programmed cell death after gamma-irradiation [10] and that mutation of abl-1/abl1 induces more germline apoptosis than wild type. Furthermore, it has been shown that loss-of-function of $c e d-9$, a BCL-2 homolog in C. elegans [11], activates CED-3 to induce programmed cell death $[12,13]$ and that the ced-9(n1653) mutant exhibits more apoptotic cells upon DNA damage treatment. Our epistasis analysis revealed that 
$n h r-14 / \mathrm{HNF} 4 \alpha$ deletion abrogated DNA damage-induced apoptosis in akt-1(ok525) (Figure 2A) but not decrease apoptosis in the ced-9(n1653) background (Figure 2D). Knockdown of $n h r-14 / \mathrm{HNF} 4 \alpha$ led to dramatically decreased germline apoptosis in abl-1(ok171) mutants (Figure 2B).

In addition, because $b r c-1$ is the BRCA1 homolog in C. elegans and functions in DNA double-strand break (DSB) repair after gamma-irradiation[14, 15], mutation of brc-1/BRCA1 resulted in failing to repair the DSB and induced apoptosis. We also found that the brc-1(tm1145);nhr-14(tm1473) double mutant dramatically decreased apoptosis compared to brc-1(tm1145) alone after DNA damage (Figure 2C).

Taken together, these findings indicate that $n h r-14 / \mathrm{HNF} 4 \alpha$ is a key regulator of DNA damage-induced programmed cell death.

\section{$n h r-14 / \mathrm{HNF} 4 \alpha$ does not affect physiological programmed cell death}

Since $n h r-14(t m 1473)$ showed less apoptosis upon gamma-irradiation, we next investigated the underlying cellular mechanism. We performed the time lapse phenotype analysis and found that there was no germline development defect and $n h r-14(t m 1473)$ showed the same apoptosis number as $\mathrm{N} 2$ at any time. These data indicate the decreased programmed cell death in $n h r-14(t m 1473)$ is neither due to germline development nor the delayed cell death. We further examined whether $n h r-14$ affects the physiological programmed cell death in embryos. Figure 3A shows that there was no difference in the number of cell apoptosis in embryos between N2 and nhr-14(tm1473). ced-1(e1735)[16] and vps-18(tm1125)[17] has been reported to affect cell corpse clearance, we also found no difference in the number of cell apoptosis in germline between wild type and $n h r-14(t m 1473)$ mutants in the background of ced-1(e1735) and vps-18(tm1125) (Figure 3B, C). In order to further prove that $n h r-14$ does not affect germline physiological programmed cell death, we analyzed the expression difference of ced-3, ced-4 and ced-9 in N2 and nhr-14(tm1473) by northern blot, our results showed that nhr-14 did not affect the mRNA levels of these three genes (Figure 3D). These results indicate that $n h r-14 / \mathrm{HNF} 4 \alpha$ only affects the DNA damage-induced apoptosis, but not the physiological programmed cell death. 
213 Previous studies demonstrated that the checkpoint signaling pathways are activated upon

214 DNA damage and play the critical role in repairing the damaged DNA or inducing

215 programmed cell death $[18,19]$. Mutations in checkpoint genes can restrain both DNA

216 damage-induced cell cycle arrest and apoptosis upon gamma-irradiation in C. elegans[18 ]. To

217 determine whether $n h r-14 / \mathrm{HNF} 4 \alpha$ is a checkpoint gene, we first assessed the sensitivity of

$218 n h r-14(t m 1473)$ mutants to gamma-irradiation using the radiation sensitivity assay. We found

219 that the survival rate of $n h r-14(\operatorname{tm} 1473)$ progeny was comparable to that of wild-type animals,

220 but was much higher than that of checkpoint gene mutants hus-1(op244) and clk-2(mn159)

221 (Table 1). In addition, $n h r-14($ tm1473) worms displayed similar cell cycle arrest in germline

222 mitotic region to that in wild type following irradiation treatment (Figure 4A). We further

223 made hus-1(op244); nhr-14(tm1473) and clk-2(mn159);nhr-14(tm1473) double mutants, and

224 found that these double mutants exhibited the same phenotype as the check point mutants

225 (Figure 4B). Therefore, $n h r-14 / \mathrm{HNF} 4 \alpha$ is not a checkpoint gene and may function

226 downstream of the checkpoint pathway.

To determine whether $n h r-14 / \mathrm{HNF} 4 \alpha$ is involved in DNA repair, we irradiated worms containing the hus-1::gfp transgene in the nhr-14(tm1473) background. We found that relocalization of HUS-1::GFP was independent of $n h r-14 / \mathrm{HNF} 4 \alpha$ (Supplemental Figure 1A), and the number of foci in $n h r-14(\operatorname{tm} 1473)$ was the same as wild type N2 (Supplemental Figure 1B). These results indicates that $n h r-14$ is necessary for irradiation induced apoptosis, but not for irradiation induced cell cycle arrest (Figure 4) and DNA repair. Our findings suggest that $n h r-14 / \mathrm{HNF} 4 \alpha$ is not involved in DNA repair and acts downstream of the checkpoint genes.

NHR-14 cooperates with CEP-1/p53 to regulate egl-1 and ced-13 transcription upon DNA damage

To investigate how $n h r-14 / \mathrm{HNF} 4 \alpha$ regulates DNA damage-induced programmed cell death, we first examined the expression level of apoptotic initiator gene egl-1 and ced-13 in N2 and $n h r-14(t m 1473)$ worms. We irradiated N2 and $n h r-14(t m 1473)$ young adult worms at a dose 
of $120 \mathrm{~Gy}$ and performed RT-qPCR experiment, our results show that gamma-irradiation-induced egl-1 and ced-13 levels were significantly reduced in nhr-14(tm1473). In N2 worms, egl-1 level was increased by 20 folds, however, in $n h r-14($ tm1473) egl-1 expression only increased 8 folds after DNA damage (Figure 5A). ced-13 expression level was induced more than 5-fold in $\mathrm{N} 2$ worms upon gamma-irradiation, but only about 3-fold in $n h r-14(\operatorname{tm} 1473)$ worms (figure 5B). These results suggest that $n h r-14 / \mathrm{HNF} 4 \alpha$ regulates DNA damage-induced $e g l-1$ and $c e d-13$. To examine if nhr-14(tm1473) affect CEP-1 level, we performed western blotting to test CEP-1 level in N2 and $n h r-14(t m 1473)$, we found that $n h r-14(t m 1473)$ did not affect the protein level of CEP-1(Figure 5C).

Previous studies demonstrated that CEP-1/p53 is a key transcription factor of $e g l-1$ and ced-13[19,20]. Because $n h r-14 / \mathrm{HNF} 4 \alpha$ regulates egl-1 and ced-13 at mRNA levels, we hypothesized that NHR-14/HNF4 $\alpha$ could be a cofactor of CEP-1/p53. To this end, we first examined if NHR-14/HNF4 $\alpha$ forms a complex with CEP-1/p53. Flag-tagged NHR-14 and Myc-CEP-1 were co-transfected into 293T cells, and then CoIP was performed. Figure 5D shows that Myc-CEP-1/p53 was co-immunoprecipitated with Flag-NHR-14, suggesting that these two proteins interact each other in mammalian cells. To investigate if NHR-14/HNF4 $\alpha$ directly binds to CEP-1/p53, we performed in vitro GST-pull down assay. We found that GST-CEP-1 fusion protein, but not GST, pulled-down ${ }^{[35 \mathrm{~S}]}$ methionine labeled NHR-14 (Figure 5E). These data suggests that NHR-14/HNF4 $\alpha$ and CEP-1/p53 might directly interact each other.

We next investigated whether $n h r-14 / \mathrm{HNF} 4 \alpha$ regulates DNA damage-induced programmed cell death through cep-1/p53. As GLD-1 is a translational repressor of cep-1/p53 via directly binding to the 3'UTR of cep-1/p53 mRNA[21], gld-1(op236) loss-of-function mutants expresses higher level of CEP-1/p53 in C. elegans. We found that egl-1 and ced-13 mRNA levels were much higher in gld-1(op236) mutants than N2 worms after gamma-irradiation (Figure 5A, B). We also demonstrated that up-regulated CEP-1/p53 in gld-1(op236) could rescue DNA damage-induced egl-1 and ced-13 expression and apoptosis (Figure 5A, B and F). 
271 cep-1/p53 and regulates DNA damage-induced programmed cell death through

272 CEP-1/p53 (Figure 5G).

273

274

275 


\section{Discussion:}

DNA damage-induced programmed cell death is associated with various human malignancies and identification of regulators in DNA damage-induced apoptosis pathway is critical for intervention of these diseases. C. elegans has been shown to be an excellent model to study DNA damage-induced programmed cell death. And thus it is very helpful for us to understanding the mechanism of carcinogenesis by studying the regulation of DNA damage-induced apoptosis in C. elegans germline.

P53 is a key tumor suppressor and its mutations were detected in more than $50 \%$ of human cancers. In C.elegans, the p53 homolog CEP-1 acts as a key effector to mediate germ cell apoptosis triggered by ionizing irradiation[22]. Identification of new co-factors of CEP-1/p53 in C.elegans may offer critical targets for cancer intervention.

In response to DNA damage stimuli, the checkpoint genes will sense the signals and induce cell cycle arrest or programmed cell death. Simultaneously, CEP-1/p53 is activated and subsequently induces up-regulation of $\mathrm{BH} 3$ genes egl-1 and ced-13. Mutation of the checkpoint genes block the transfer of DNA damage signals and reduce DNA damage-induced apoptosis. Nuclear hormone receptor family is a key to many important cellular processes, but the role of NHR family in DNA damage-induced programmed cell death remains elusive. Previous study showed that NHR-14/HNF4 $\alpha$, which was thought to be an estrogenic hormone receptor[9], was involved in the immune response processes via regulation of vitellogenin expression[23]. In present report, we identified $n h r-14$ /HNF4 $\alpha$ as an important member of NHR in regulation of DNA damage-induced apoptosis. Moreover, we showed that $n h r-14 / \mathrm{HNF} 4 \alpha$ is primarily involved in regulation of the DNA damage-induced apoptosis, but not the physiological programmed cell death (Figure 3). Mechanically, our experiment revealed that $n h r-14$ /HNF4 $\alpha$ deletion decreases DNA damage-induced up-regulation of egl-1 and ced-13. More significantly, we showed that NHR-14/HNF4 $\alpha$ interacts with CEP-1/p53 and functions as a cofactor of CEP-1/p53. In addition, $n h r-14(t m 1473)$ mutant dramatically reduces CEP-1/p53-mediated DNA damage-induced apoptosis. Thus we consider $n h r-14$ is a general positive regulator of DNA damage-induced germline apoptosis. Our study first reported a nuclear hormone receptor 
NHR-14/HNF4 $\alpha$ that is involved in DNA damage-induced apoptosis . Identification of NHR-14/HNF4 $\alpha$ interaction with CEP-1/p53 to control DNA damage-induced egl-1 and ced-13 could provide new targets for cancer intervention.

Dysregulation of DNA damage induced apoptosis usually leads to tumorigenesis. Next, we will further confirm the relationship between the dysregulation of DNA damage induced apoptosis by nhr-14/HNF4 $\alpha$ deletion and tumorigenesis and will further study the mechanism of HNF $4 \alpha$ in tumorigenesis.

\section{Conclusions}

313 Our study revealed the function of NHR-14 in DNA damage induced apoptosis. $314 n h r-14 / \mathrm{HNF} 4 \alpha$ functions as a cofactor of cep-1/p53 to regulate DNA damage-induced programmed cell death.

\section{Abbreviations}

NHR, Nuclear hormone receptors; HNF4, hepatocyte nuclear factor; PRMT, protein arginine methyltransferase; UTR, Untranslated Regions; PMSF, phenylmethylsulfonyl fluoride; SDS-PAGE, sodium dodecyl sulfate polyacrylamide gel electrophoresis

\section{Declarations}

Ethical Approval and Consent to participate

Not applicable

\section{Consent for publication}

Not applicable

Availability of supporting data

Not applicable

Competing interests

The authors declare no competing interests.

\section{Acknowledgements}

331 We thank C. elegans Genetic Center and Dr. Shohei Mitani for providing C.elegans deletion strains. We especially thank Dr. Chonglin Yang for helpful suggestion, reagents and the comments on the manuscript. 


\section{Funding}

335 This work was supported by Applied Basic Research Foundation of Yunnan Province (Grant

336 No. 202101AV070002 and 2019FY003030) and Major Science and Technique Programs in

337 Yunnan Province (Grant No. 202102AA310055)

338 Authors' information

339 Center for Life Sciences, School of Life Sciences, State Key Laboratory for Conservation and

340 Utilization of Bio-Resources in Yunnan, Yunnan University, Kunming, China

341 Lei Sang, Rui Dong, Qinggang Hao, Mei Yang, Jianwei Sun

342 Clinical Laboratory, the second Affiliated Hospital of Kunming Medical University,

343 Kunming, China

344 Rui Liu

345 The Sixth Affiliated Hospital of Kunming Medical University, Yuxi, China

346 Xingshun Wang, Yong Zeng

347 Authors' Contributions

348 JWS and MY designed the study. JWS and MY wrote the manuscript text. LS, RD and RL

349 conducted experiments, and the other authors took part in literature collection and data

350 analysis as assistants. All authors read and approved the final manuscript.

\section{Corresponding author}

352 Correspondence to $\underline{\text { Jianwei Sun }}$ or Mei Yang

353

354

355 


\section{Reference}

1. Antebi, A., Nuclear hormone receptors in C. elegans. WormBook, 2006: p. 1-13.

2. Magner, D.B., et al., The NHR-8 nuclear receptor regulates cholesterol and bile acid homeostasis in C. elegans. Cell Metab, 2013. 18(2): p. 212-24.

3. Verghese, E., et al., The tailless ortholog nhr-67 functions in the development of the C. elegans ventral uterus. Dev Biol, 2011. 356(2): p. 516-28.

4. Goudeau, J., et al., Fatty acid desaturation links germ cell loss to longevity through NHR-80/HNF4 in C. elegans. PLoS Biol, 2011. 9(3): p. e1000599.

5. Liang, B., et al., The role of nuclear receptor NHR-64 in fat storage regulation in Caenorhabditis elegans. PLoS One, 2010. 5(3): p. e9869.

6. Rajan, M., et al., NHR-14 loss of function couples intestinal iron uptake with innate immunity in C. elegans through PQM-1 signaling. eLife Sciences, 2019. 8.

7. Yang, M., et al., Caenorhabditis elegans protein arginine methyltransferase PRMT-5 negatively regulates DNA damage-induced apoptosis. PLoS Genet, 2009. 5(6): p. e1000514.

8. Schumacher, B., et al., The C. elegans homolog of the 553 tumor suppressor is required for DNA damage-induced apoptosis. Curr Biol, 2001. 11(21): p. 1722-7.

9. Mimoto, A., et al., Identification of an estrogenic hormone receptor in Caenorhabditis elegans. Biochem Biophys Res Commun, 2007. 364(4): p. 883-8.

10. Quevedo, C., D.R. Kaplan, and W.B. Derry, AKT-1 regulates DNA-damage-induced germline apoptosis in C. elegans. Curr Biol, 2007. 17(3): p. 286-92.

11. Hengartner, M.O. and H.R. Horvitz, C. elegans cell survival gene ced-9 encodes a functional homolog of the mammalian proto-oncogene bcl-2. Cell, 1994. 76(4): p. 665-76.

12. Seshagiri, S. and L.K. Miller, Caenorhabditis elegans CED-4 stimulates CED-3 processing and CED-3-induced apoptosis. Curr Biol, 1997. 7(7): p. 455-60.

13. Spector, M.S., et al., Interaction between the C. elegans cell-death regulators CED-9 and CED-4. Nature, 1997. 385(6617): p. 653-6.

14. Adamo, A., et al., BRC-1 acts in the inter-sister pathway of meiotic double-strand break repair. EMBO Rep, 2008. 9(3): p. 287-92.

15. Boulton, S.J., et al., BRCAl/BARD1 orthologs required for DNA repair in Caenorhabditis elegans. Curr Biol, 2004. 14(1): p. 33-9.

16. Zhou, Z., E. Hartwieg, and H.R. Horvitz, CED-1 is a transmembrane receptor that mediates cell corpse engulfment in C. elegans. Cell, 2001. 104(1): p. 43-56.

17. Xiao, H., et al., Lysosome biogenesis mediated by vps-18 affects apoptotic cell degradation in Caenorhabditis elegans. Mol Biol Cell, 2009. 20(1): p. 21-32.

18. Gartner, A., et al., A conserved checkpoint pathway mediates DNA damage--induced apoptosis and cell cycle arrest in C. elegans. Mol Cell, 2000. 5(3): p. 435-43.

19. Hofmann, E.R., et al., Caenorhabditis elegans HUS-1 is a DNA damage checkpoint protein required for genome stability and EGL-1-mediated apoptosis. Curr Biol, 2002. 12(22): p. 1908-18.

20. Schumacher, B., et al., C. elegans ced-13 can promote apoptosis and is induced in response to DNA damage. Cell Death Differ, 2005. 12(2): p. 153-61.

21. Schumacher, B., et al., Translational repression of C. elegans p53 by GLD-1 regulates DNA damage-induced apoptosis. Cell, 2005. 120(3): p. 357-68.

22. Han, Z., et al., The C. elegans Tousled-like kinase contributes to chromosome segregation as a 
400

401

402

403

404

405

406 substrate and regulator of the Aurora B kinase. Curr Biol, 2005. 15(10): p. 894-904.

23. Fischer, M., et al., Phytoestrogens genistein and daidzein affect immunity in the nematode Caenorhabditis elegans via alterations of vitellogenin expression. Mol Nutr Food Res, 2012. 56(6): p. 957-65. 
Figure 1. Inactivation of $n h r-14 / \mathrm{HNF} 4$ inhibits DNA damage-induced programmed cell death in prmt-5(gk357)

A. Quantitative analysis of germ cell apoptosis in control RNAi- and nhr-14 RNAi-treated N2 and prmt-5(gk357) animals. N2 and prmt-5(gk357) were fed with control RNAi and

Figure 2. Epistasis analysis indicates $n h r-14 / \mathrm{HNF} 4$ mediating DNA damage-induced apoptosis

A. Quantitative analysis germ cell apoptosis induced by gamma-irradiation in N2, nhr-14(tm1473), akt-1(ok525) and akt-1(ok525); nhr-14(tm1473). Young adult animals were irradiated with gamma-ray (120 Gy) and analyzed at indicated time points after irradiation. Error bars represent standard error of the mean (SEM).

B. Quantitative analysis germ cell apoptosis in control RNAi and $n h r-14$ RNAi-treated N2 and $a b l-1(o k 171)$ animals. N2 and $a b l-1(o k 171)$ were fed with control RNAi and $n h r-14$ 
RNAi and then (L4) was irradiated at 120Gy. After 36 hours of gamma-irradiation, germ cell apoptosis from one gonad arm of each animal were scored from at least 20 animals. Error bars represent standard error of the mean (SEM).

C. Quantitative analysis of germ cell apoptosis induced by gamma-irradiation in N2, $n h r-14(t m 1473), b r c-1(t m 1145)$ and $b r c-1(t m 1145) ; n h r-14(t m 1473)$ animals.

D. Quantitative analysis germ cell apoptosis induced by gamma-irradiation in N2, nhr-14(tm1473), ced-9(n1653) and ced-9(n1653); nhr-14(tm1473) animals.

Figure 3. nhr-14 doesn't affect the physiological programmed cell death

A. Quantification of embryo cell apoptosis in N2 and $n h r-14(t m 1473)$ mutants.

B. Quantification of germline cell apoptosis in ced-1(e1375) and ced-1(e1375); $n h r-14($ tm1473)animals at indicated time points post L4.

C. Quantification of germline cell apoptosis in vps-18(tm1125) and vps-18(tm1125);

Figure 4. $n h r-14$ is not a checkpoint gene and does not affect the cell cycle arrest after DNA damage

A. Representative image of DAPI staining of the germline mitotic region in N2, nhr-14(tm1473) and hus-1(op234) worms. Young adult worms were treated with gamma-irradiation at $120 \mathrm{~Gy}$. After 36 hours of irradiation, germline was dissected and stained with DAPI. Bars, $5 \mu \mathrm{m}$.

B. Quantification of germline mitotic nuclear number after gamma-irradiation in N2, 
Figure 5. NHR-14 cooperates with CEP-1/p53 to regulate egl-1 and ced-13 expression

A. Relative fold induction of egl-1 mRNA in N2, nhr-14(tm1473), gld-1(op236) and

E. NHR-14 and CEP-1/p53 directly interact in vitro. The full-length NHR-14 protein was in vitro translated and labeled with $\left[{ }^{35} \mathrm{~S}\right]$ methionine and incubated with GST or GST-CEP-1/p53 fusion proteins, which were immobilized on glutathione sepharose beads, for $2 \mathrm{~h}$. After extensive washes, the bound proteins were resolved by SDS-PAGE and detected by autoradiography

F. Quantitative analysis germ cell apoptosis induced by gamma-irradiation at indicated time points post L4 in N2, nhr-14(tm1473), gld-1(op236) and gld-1(op236);nhr-14(tm1473). Up-regulated CEP-1/p53 via loss function of gld-1(op236) rescued DNA damage-induced programmed cell death in $n h r-14(\mathrm{tm} 1473)$ animals.

G. The genetic pathway for $n h r-14 / \mathrm{HNF} 4$ to regulate DNA damage-induced apoptosis and hepatocarcinogenesis. NHR-14/HNF4 functions as a cofactor of CEP-1/p53 to regulate DNA damage induced apoptosis via controlling egl-1 and ced-13 expression. 
DNA damage. The arrows indicate HUS-1::GFP foci.

498 B. Quantification of germline HUS-1:GFP foci in N2 and $n h r-14(t m 1473)$ before and after DNA damage. N2 and $n h r-14(t m 1473)$ L4 worms were irradiated. After 36 hours of gamma-irradiation, the number of HUS-1:GFP foci from one gonad arm of each animal were scored from at least 20 animals. Error bars represent standard error of the mean (SEM).

503 
506 Table 1 nhr-14 does not affect the survival of progeny after gamma-irradiation

507 treatment

508 The Survival of $n h r-14(t m 1473)$ Mutant Progeny Is Not Sensitive to Irradiation

\begin{tabular}{|c|c|c|c|c|c|c|}
\hline irradiatio & \multicolumn{6}{|c|}{ Survival(\%) } \\
\hline \multirow[t]{2}{*}{ Dose(Gy) } & $N 2$ & $n h r-14(\mathrm{tm} 147$ & hus-1(op24 & hus-1;nhr- & clk-2(mn15 & clk-2;nhr-1 \\
\hline & & 3) & 4) & 14 & 9) & 4 \\
\hline \multirow[t]{2}{*}{0} & $100.0 \pm$ & $100.0 \pm 0$ & $97.6 \pm 0.1$ & $98.3 \pm 0.8$ & $98.0 \pm 0.7$ & $96.0 \pm 1.5$ \\
\hline & $\mathbf{0}$ & & & & & \\
\hline \multirow[t]{2}{*}{40} & $86.3 \pm 1$. & $86.1 \pm 1.8$ & $37.7 \pm 1.1$ & $39.6 \pm 2.0$ & $29.1 \pm 2.6$ & $28.9 \pm 1.7$ \\
\hline & 2 & & & & & \\
\hline \multirow[t]{2}{*}{80} & $76.7 \pm 2$. & $76.3 \pm 1.8$ & $18.7 \pm 1.3$ & $18.0 \pm 1.2$ & $9.4 \pm 0.5$ & $11.1 \pm 0.8$ \\
\hline & 4 & & & & & \\
\hline \multirow[t]{2}{*}{120} & $64.5 \pm 1$. & $62.5 \pm 1.4$ & $3.2 \pm 0.5$ & $3.5 \pm 0.7$ & $2.8 \pm 1.0$ & $2.8 \pm 1.0$ \\
\hline & 6 & & & & & \\
\hline
\end{tabular}

509 


\section{Figures}

\section{Figure 1}

Inactivation of nhr-14/HNF4 inhibits DNA damage-induced programmed cell death in prmt-5(gk357)

A. Quantitative analysis of germ cell apoptosis in control RNAi- and nhr-14 RNAi-treated N2 and prmt-5(gk357) animals. N2 and prmt-5(gk357) were fed with control RNAi and nhr-14 RNAi and then (L4) was irradiated. After 36 hours of gamma-irradiation, germ cell apoptosis from one gonad arm of each animal were scored from at least 20 animals..

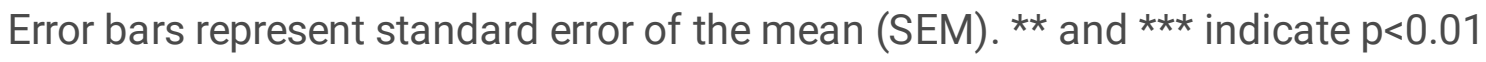
and 0.001 , respectively.

B. Quantitative analysis germ cell apoptosis induced by gamma-irradiation in N2, nhr-14(tm1473), prmt-5(gk357) and prmt-5(gk357); nhr-14(tm1473). Germ cell apoptosis from one gonad arm of each animal were scored after $36 \mathrm{~h}$ of irradiation at indicated doses. At least 20 worms were scored at each radiation dose or time point. Error bars represent standard error of the mean (SEM).

C. Quantitative analysis germ cell apoptosis at indicated time points after irradiation (120 Gy) in N2, nhr-14(tm1473), prmt-5(gk357) and prmt-5(gk357); nhr-14(tm1473) animals.

D. Quantitative analysis of germ cell apoptosis in ced-1(e1375); prmt-5(gk357) and ced-1(e1375); prmt-5(gk357); nhr-14(tm1473) aninmals with and without IR treatment. $\star \star \star$ indicate $p<0.001$ 
Epistasis analysis indicates nhr-14/HNF4 mediating DNA damage-induced apoptosis

A. Quantitative analysis germ cell apoptosis induced by gamma-irradiation in N2, nhr-14(tm1473), akt-1(ok525) and akt-1(ok525); nhr-14(tm1473). Young adult animals were irradiated with gamma-ray (120 Gy) and analyzed at indicated time points after irradiation. Error bars represent standard error of the mean (SEM).

B. Quantitative analysis germ cell apoptosis in control RNAi and nhr-14 RNAi-treated N2 and abl-1(ok171) animals. N2 and abl-1(ok171) were fed with control RNAi and nhr-14 RNAi and then (L4) was irradiated at 120Gy. After 36 hours of gamma-irradiation, germ cell apoptosis from one gonad arm of each animal were scored from at least 20 animals. Error bars represent standard error of the mean (SEM).

C. Quantitative analysis of germ cell apoptosis induced by gamma-irradiation in N2, nhr-14(tm1473), brc-1(tm1145) and brc-1(tm1145);nhr-14(tm1473) animals.

D. Quantitative analysis germ cell apoptosis induced by gamma-irradiation in N2, nhr-14(tm1473), ced-9(n1653) and ced-9(n1653); nhr-14(tm1473) animals.

\section{Figure 3}

nhr-14 doesn't affect the physiological programmed cell death

A. Quantification of embryo cell apoptosis in N2 and nhr-14(tm1473) mutants.

B. Quantification of germline cell apoptosis in ced-1(e1375) and ced-1(e1375); nhr-14(tm1473)animals at indicated time points post L4.

C. Quantification of germline cell apoptosis in vps-18(tm1125) and vps-18(tm1125); nhr-14(tm1473) animals at indicated time points post $L 4$. 
D. Northern blot analysis ced-3, ced-4 and ced-9 mRNA level in N2 and nhr-14(tm1473) at 36 hours post L4.

\section{Figure 4}

nhr-14 is not a checkpoint gene and does not affect the cell cycle arrest after DNA damage

A. Representative image of DAPI staining of the germline mitotic region in N2, nhr-14(tm1473) and hus-1(op234) worms. Young adult worms were treated with gamma-irradiation at $120 \mathrm{~Gy}$. After 36 hours of irradiation, germline was dissected and stained with DAPI. Bars, $5 \mu \mathrm{m}$.

B. Quantification of germline mitotic nuclear number after gamma-irradiation in N2, nhr-14(tm1473), hus-1(op244), hus-1(op234);nhr-14(tm1473), clk-2(mn159), clk-2(mn159);nhr-14 (tm1473) worms. The gray and black bars represent nuclei number in mitotic region in control and gamma irradiation-treated worm germline, respectively. ${ }^{\star \star \star}$ indicate $p<0.001$

\section{Figure 5}

NHR-14 cooperates with CEP-1/p53 to regulate egl-1 and ced-13 expression A. Relative fold induction of egl-1 mRNA in N2, nhr-14(tm1473), gld-1(op236) and gld-1(op236);nhr-14(tm1473) after 24 hours of gamma-irradiation (120 Gy). egl-1 fold induction was averaged from three independent RT-qPCR analyses.

B. Relative fold induction of ced-13 mRNA in N2, nhr-14(tm1473), gld-1(op236) and gld-1(op236);nhr-14(tm1473) following 24 hours of gamma-irradiation (120 Gy). egl-1 fold change was averaged from three independent RT-qPCR analyses. 
C. Western blotting analysis of CEP-1 level in N2 and nhr-14(tm1473) animals

D. NHR-14 interacts with CEP-1/p53 in mammalian cells. Flag-NHR-14 and Myc-CEP-1/p53 were co-expressed in HEK293 cells and then immunoprecipitated (IP) using Flag antibody. The immunoprecipitated proteins were detected by immuno-blotting (IB) with Myc antibody.

E. NHR-14 and CEP-1/p53 directly interact in vitro. The full-length NHR-14 protein was in vitro translated and labeled with [35 481 S] methionine and incubated with GST or GST-CEP-1/p53 fusion proteins, which were immobilized on glutathione sepharose beads, for $2 \mathrm{~h}$. After extensive washes, the bound proteins were resolved by SDS-PAGE and detected by autoradiography

F. Quantitative analysis germ cell apoptosis induced by gamma-irradiation at indicated time points post L4 in N2, nhr-14(tm1473), gld-1(op236) and gld-1(op236);nhr-14(tm1473). Up-regulated CEP-1/p53 via loss function of gld-1(op236) rescued DNA damage-induced programmed cell death in nhr-14(tm1473) animals.

G. The genetic pathway for nhr-14/HNF4 to regulate DNA damage-induced apoptosis and hepatocarcinogenesis. NHR-14/HNF4 functions as a cofactor of CEP-1/p53 to regulate DNA damage induced apoptosis via controlling egl-1 and ced-13 expression.

\section{Supplementary Files}

This is a list of supplementary files associated with this preprint. Click to download.

- supplementalfigure.tif 